\title{
LETTER
}

\section{Hypernatremia and intracranial pressure: more questions than answers}

\author{
Donald E G Griesdale*1-3, Mypinder S Sekhon' and William R Henderson' \\ See related research by Wells et al., http://ccforum.com/content/16/5/R193
}

\begin{abstract}
The observational literature suggests that hypernatremia is associated with worse outcomes in patients with traumatic brain injury. In a previous issue of Critical Care, Wells and colleagues add to this literature by failing to show an association between hypernatremia and reduced intracranial pressure. However, we must bear in mind many limitations of observational methods before eliminating hyperosmolar therapy from our armamentarium.
\end{abstract}

In a previous issue of Critical Care, Wells and colleagues [1] report on their retrospective cohort study examining the relationship between serum sodium and intracranial pressure (ICP) with severe traumatic brain injury (TBI). This is an ongoing topic of interest to neurointensivists as hyperosmolar therapy remains a treatment option for elevated ICP [2]. We commend the authors for their contribution to a fundamentally important issue in neurocritical care; however, several issues deserve further discussion.
A critical limitation of observational methodology is confounding by indication, which exists when variables associated with exposure are also associated with outcomes in the study base [3]. Clinicians administer hyperosmolar therapy and induce hypernatremia on the basis of measured and unmeasured characteristics of patients. Lack of a formalized TBI protocol, including indications for hypertonic saline (HTS), can exacerbate this bias. For example, clinicians in the study by Wells and colleagues used both boluses and infusions of HTS with a range of administered concentrations ( $3 \%$ versus $7.5 \%$ ). No attempt was made to adjust for differences in baseline characteristics of patients. Furthermore, although regression is an important method to help reduce bias, several assumptions must be met for the analysis to be valid. Linear regression is predicated upon independence of data. With 1,230 paired sodium and ICP measurements in 81 patients, there is likely to be marked within-subject correlation of data that is not taken into account by this analysis. Consequently, a linear mixed model or other analysis is warranted to specifically model and account for this correlation [4]. Failing to do so limits the interpretability of their results.

\section{Authors' response}

Diana L Wells, Joseph M Swanson, G Christopher Wood, Louis J Magnotti, Bradley A Boucher, Martin A Croce, Charles G Harrison, Michael S Muhlbauer and Timothy C Fabian

We thank Griesdale and colleagues for their thoughtful letter describing concerns with our study, specifically the retrospective design and statistical tests. Owing to the lack of literature supporting the benefits of HTS to induce hypernatremia in patients with TBI, we pragmatically

\footnotetext{
${ }^{*}$ Correspondence: donald.griesdale@vch.ca

'Department of Medicine, Division of Critical Care Medicine, Vancouver General

Hospital, University of British Columbia, 855 West 12th Avenue, Vancouver, BC,

V5Z 1M9, Canada

Full list of author information is available at the end of the article
}

evaluated this practice in one of the largest studies to date $[1,5,6]$.

We agree with the limitations mentioned by Griesdale and colleagues in regard to the lack of a formalized TBI protocol and the inclusion of various doses of HTS, and we addressed this in our discussion. Importantly, patients in our study were treated with the same general approach to hyperosmolar therapy ( $84 \%$ of patients received $3 \%$ $\mathrm{NaCl}$ boluses) [1].

In regard to adjustment for baseline characteristics, we performed linear regression in important subgroups divided by baseline ICP and Glasgow Coma Scale score. 
Furthermore, to account for within-subject correlation of data, we conducted linear regression for each individual patient as described in Table 2 of the article. In none of the many analyses performed was there an indication of a general correlation between serum sodium and ICP [1]. Nonetheless, we agree that other statistical methods such as a linear mixed model could have possibly shed more light on these data.

Recognizing the limitations of our study is important and should serve as a call to the medical community for the need of a well-designed, prospective randomized controlled trial evaluating the use of HTS to induce hypernatremia in TBI. This is essential before we can truly include or limit this therapy as a valid option in our treatment armamentarium for these patients.

Abbreviations

HTS, hypertonic saline; ICP, intracranial pressure; TBI, traumatic brain injury.

\section{Competing interests}

The authors declare that they have no competing interests.

\section{Author details}

'Department of Medicine, Division of Critical Care Medicine, Vancouver General Hospital, University of British Columbia, 855 West 12th Avenue Vancouver, BC, V5Z 1M9, Canada. ${ }^{2}$ Department of Anesthesia, Pharmacology and Therapeutics and Department of Medicine, Division of Critical Care
Medicine, Vancouver General Hospital, University of British Columbia, 855 West 12th Avenue, Vancouver, BC, V5Z 1M9, Canada. ${ }^{3}$ Centre for Clinical Epidemiology and Evaluation, Vancouver Coastal Health Research Institute, 828 West 10 th Avenue, Vancouver, BC, V5Z 1M9, Canada.

Published: 7 January 2013

\section{References}

1. Wells DL, Swanson JM, Wood GC, Magnotti $\amalg$, Boucher BA, Croce MA, Harrison CG, Muhlbauer MS, Fabian TC: The relationship between serum sodium and intracranial pressure when using hypertonic saline to target mild hypernatremia in patients with head trauma. Crit Care 2012, 16:R193.

2. Helmy A, Vizcaychipi M, Gupta A: Traumatic brain injury: intensive care management. Br J Anaesth 2007, 99:32-42.

3. Salas $M$, Hofman $A$, Stricker $B H$ : Confounding by indication: an example of variation in the use of epidemiologic terminology. Am J Epidemiol 1999, 149:981-983.

4. Mascha EJ, Sessler DI: Equivalence and noninferiority testing in regression models and repeated-measures designs. Anesth Analg 2011, 112:678-687.

5. Shackford SR, Bourguignon PR, Wald SL, Rogers FB, Osler TM, Clark DE: Hypertonic saline resuscitation of patients with head injury: a prospective, randomized clinical trial. J Trauma 1998, 44:50-58.

6. Qureshi Al, Suarez JI, Castro A, Bhardwaj A: Use of hypertonic saline/acetate infusion in treatment of cerebral edema in patients with head trauma: experience at a single center. J Trauma 1999, 47:659-665.

doi:10.1186/cc11888

Cite this article as: Griesdale DEG, et al:. Hypernatremia and intracranial pressure: more questions than answers. Critical Care 2013, 17:4010. 